\title{
Adaptive Discrete-Time Sliding Mode Control of Infinite-Dimensional Systems.
}

\author{
Vadim Utkin \\ Electrical Engineering Department \\ The Ohio State University \\ 2015 Neil Avenue \\ Columbus, Ohio 43210 \\ E-mail utkin@ee.eng.ohio-state.edu
}

\begin{abstract}
The theory of sliding mode control has been mainly developed for finite-dimensional continuous-time systems governed by ordinary differential equations. The concept is generalized for discrete-time implementation in infinite-dimensional systems differential equations in Banach spaces. The distributed parameter identification method is developed for thermal processes along with an adaptive discrete-time control algorithm.
\end{abstract}

\section{Introduction}

The sliding mode control methodology implies decoupling a design problem into two independent subproblems of lower dimensions: design of the desired dynamics in sliding mode and enforcing this motion in some manifold in the system state $[1,2,3]$. The main obstacle for application of the control methods for infinite-dimensional systems is unboundness of operators in motion equations. Recently several papers have been published on mathematical aspects and design methods of sliding mode control in distributed parameter systems governed by partial differential equations and ordinary differential equations in Banach spaces [4.5.6]. It was shown that for the systems with elliptic operators the equivalent control method, developed for finitedimensional systems, may be used for deriving sliding mode equations and the desired dynamics of this motion may be designed by a proper choice of the discontinuity manifold.

The sliding mode is enforced by means of discontinuities in control on some manifold in the system state space. The discontinuity manifold $\mathrm{S}$, containing state trajectories is attained after a finite time interval. From mathematical point of view it should be emphasized that the Cauchy problem does not have a unique solution for $t<0$. In other words a shift operator establishing correspondence between the state in two different time instants is not invertible in the points on the sliding manifold. Indeed any point where sliding mode exists may be reached along a sliding trajectory in $\mathrm{S}$ or by a trajectory from outside of S. For discrete-time systems the concept of sliding mode needs to be clarified since discontinuous control does not enable enforcing motion in an arbitrary manifold, and results in chattering or oscillations at the boundary layer at the sampling frequency.

In contrast to continuous-time systems, in discrete-time systems with continuous control the motion may exist with state trajectories in some manifold with a finite time interval preceding this motion. So the motion may be called "sliding mode". Similarly to continuous-time systems with sliding modes the shift operator in discretetime systems is not invertible. In discrete-time systems the continuous operator in the system equation, which matches a system state from one sampling instant into the next one, is a shift operator. If the sliding mode occurs, state trajectories are in a manifold of lower dimension than that of the original system. This means that the inverse of the shift operator does not exist since it transforms a domain of full dimension into another domain of lower dimension. The concept of discrete-time sliding mode is introduced in terms of shift operators and semigroup conditions $[7,8,9]$. In this paper the concept is generalized for discrete-time implementation in infinite-dimensional systems governed by differential equations in Banach spaces. The distributed parameter identification method is developed for thermal processes along with an adaptive sliding mode control algorithm.

\section{Discrete-Time Control}

First we will outline the discrete-time sliding mode control design methodology for finite-dimensional systems

$\dot{x}=A x+B u$

where $x \in R^{n}, u \in R^{m}, A$ and $B$ are constant matrices. For a discrete-time controller with control $u$ being 
constant within a sampling interval $\delta$, the motion equations may be written as

$x_{k+1}=A^{*} x_{k}+B^{*} u_{k}$,

$x_{k}=x(k \delta), u_{k}=$ const for $k \delta \leq t<(k+1) \delta$.

$A^{*}=e^{A \delta}, \oint^{\delta} e^{A(\delta-\tau)} B d \tau$.

The control is selected such that sliding mode occurs in some manifold

$s=C x=0, s \in R^{m}, C$ is a constant matrix.

The sliding mode is governed by $(n-m)$-th order equation and the desired dynamics are assigned by a proper choice of matrix $C$. The basic idea of the control design implies selection of discontinuous control of continuous-time systems and continuous control in discrete-time systems such that the state reaches the manifold $s=0$ after finite time interval and then the state trajectories are in this manifold. For discrete-time systems the control $u_{k}$ is found such that $s_{k+1}=0$.

Then $s_{k}$ will be equal to zero identically after one step. However the control is inverse proportional to a sampling interval $\delta$ and can not be implemented in real systems with constraints posed on control actions. As it shown in [9], the motion in the manifold may be enforced after a finite number of steps in systems with bounded control. (See [9] for details of discrete-time sliding mode control design in linear finite-dimensional systems).

The design methods are developed in this paper for discrete-time sliding mode control design in infinitedimensional systems with generally speaking unbounded elliptic operator $A$ in motion equation (1). The state $x$ and control $u$ are elements of Banach spaces, and $B$ is a linear bounded operator. The control has discontinuities in some manifold

$s=C x=0$

with bounded operator $C$.

For real physical processes the solution to the differential equation with $u=0$ is bounded

$x(t)=U(t) x(0)$

where $U(t)$ is an analytical bounded semigroup, generated by operator $A[10]$.

Generalization of the discrete-time approach for infinitedimensional systems is simpler than for continuous-time ones since the operators determining the dependence between $x(t)$ and $x(t+\delta)$
$x(t+\delta)=U(\delta) x(t)+\int_{\delta}^{\delta} U(\delta-\tau) B u(\tau) d \tau$

are bounded. Correspondingly for digital control $u(t)=u_{k}=$ const for $\delta k<t<\delta(k+1)$ all the operators in discrete-time equations similar to (2) are bounded

$$
\begin{aligned}
& x_{k+1}=A^{*} x_{k}+B^{*} u_{k}, \\
& A^{*}=U(\delta), \\
& B^{*}=\int_{\delta}^{\delta} U(\delta-\tau) B d \tau .
\end{aligned}
$$

The control, reducing function $s=C x$ to zero after one step, may be found from equation

$$
\begin{aligned}
s_{k+1} & =C A^{*} x_{k}+C B^{*} u_{k} \\
& =s_{k}+\left(C A^{*}-C\right) x_{k}+C B^{*} u_{k}=0
\end{aligned}
$$

assuming that operator $\left(C B^{*}\right)$ is invertible

$$
u_{k_{e q}}=-\left(C B^{*}\right)^{-1}\left[s_{k}+\left(C A^{*}-C\right) x_{k}\right]
$$

Similarly to continuous-time systems, the continuous control enforcing motion in a sliding manifold is referred to as equivalent control. This function tends to infinity with $\delta \rightarrow 0$, since $\left\|B^{*}\right\| \rightarrow 0$ and $\left\|\left(C B^{*}\right)^{-1}\right\| \rightarrow \infty$, while $\left\|\left(C B^{*}\right)^{-1}\left(C A^{*}-C\right)\right\|$ takes finite values. It means that the bounds for the control should be taken into account.

Suppose that the control may vary within the domain $\|u\| \leq u_{0}, u_{0}$ is a constant value, and

$$
\left\|\left(C B^{*}\right)^{-1}\right\|\left\|\left(C A^{*}-C\right) x_{k}\right\|<u_{0}
$$

The control

$u_{k}=\left\{\begin{array}{l}u_{k_{e q}} \quad \text { if }\left\|u_{k_{e q}}\right\| \leq u_{0} \\ u_{0} \frac{u_{k_{e q}}}{\left\|u_{k_{e q}}\right\|} \text { if }\left\|u_{k_{e q}}\right\|>u_{0}\end{array}\right.$

is in the admissible domain.

It follows from (6-8) that for $\left\|u_{k_{e q}}\right\|>u_{0}$ 


$$
\begin{gathered}
s_{k+1}=\left[s_{k}+\left(C A^{*}-C\right) x_{k}\right]\left(1-\frac{u_{0}}{\left\|u_{k_{e q}}\right\|}\right), \\
\left(1-\frac{u_{0}}{\left\|u_{k_{e q}}\right\|}\right)>0 .
\end{gathered}
$$

Then

$$
\begin{aligned}
\left\|s_{k+1}\right\| & =\left\|s_{k}+\left(C A^{*}-C\right) x_{k}\right\|\left(1-\frac{u_{0}}{\left\|u_{k_{e q}}\right\|}\right) \\
& \leq\left\|s_{k}\right\|+\left\|\left(C A^{*}-C\right) x_{k}\right\| \\
& -\frac{u_{0}\left\|s_{k}+\left(C A^{*}-C\right) x_{k}\right\|}{\left\|\left(C B^{*}\right)^{-1}\right\|\left\|s_{k}+\left(C A^{*}-C\right) x_{k}\right\|} \\
& \leq\left\|s_{k}\right\|+\left\|\left(C A^{*}-C\right) x_{k}\right\|-\frac{u_{0}}{\left\|\left(C B^{*}\right)^{-1}\right\|} \\
& \leq\left\|s_{k}\right\| .
\end{aligned}
$$

Hence $\left\|\mathrm{S}_{k}\right\|$ decreases monotonously and according to (6), (7), after a finite number of steps, $u_{k_{e q}}$ will belong to the admissible domain, $\left\|u_{k_{e q}}\right\|<u_{0}$, and $u=u_{k_{e q}}$. According to the definition of the equivalent control, the subsequent part of the trajectory will be in manifold $s=0$ and discrete-time sliding mode will take place.

To derive the sliding mode equation in the manifold $s=0$, equivalent control (6) should be substituted for $u_{k}$ into the original system (5)

$$
x_{k+1}=\left[A^{*}-B^{*}\left(C B^{*}\right)^{-1} C A^{*}\right] x_{k}, s=0 .
$$

The desired dynamic properties of the sliding mode may be provided by a proper choice of the operator $C$.

For the control under uncertainty conditions (at the presence of disturbances or unknown parameters in the operators in motion equations) the above control is replaced by

$$
u_{k}=\left\{\begin{array}{c}
-\left(C B^{*}\right)^{-1} \mathrm{~s}_{\mathrm{k}} \text { if }\left\|\left(C B^{*}\right)^{-1} \mathrm{~s}_{\mathrm{k}}\right\| \leq u_{0} \\
-u_{0} \frac{\left(C B^{*}\right)^{-1} \mathrm{~s}_{\mathrm{k}}}{\left\|\left(C B^{*}\right)^{-1} \mathrm{~s}_{\mathrm{k}}\right\|} \text { if }\left\|\left(C B^{*}\right)^{-1} \mathrm{~s}_{\mathrm{k}}\right\|>u_{0}
\end{array} .\right.
$$

The state trajectories are not confined to the manifold $s=0$ but run in its $\delta$-vicinity and the desired sliding motion may be provided within $\delta$ accuracy [9].

To improve the accuracy of the system with unknown operator $A^{*}$, the control may be designed in the form

$u_{k_{\text {eq }}}=-\left(C B^{*}\right)^{-1}\left[s_{k}+\left(C \hat{A}^{*}-C\right) x_{k}\right]$

with $\hat{A}^{*}$ as an estimate of operator $A^{*}$.

The values of $s_{k+1}$

$s_{k+1}=-\left(C B^{*}\right)^{-1}\left(C \bar{A}^{*}-C\right) x_{k}$,

depend on the estimation error $\bar{A}^{*}=A^{*}-\hat{A}^{*}$. This function may be used for identification of operator $A^{*}$ and designing adaptive control with the trajectories tending to manifold $s=0$ or to the motion of the system with complete information.

The adaptive control may be implemented based on an identification process using the model of the process with adjustable parameters. The latter option will be demonstrated for design of discrete-time sliding mode adaptive control for a thermal distributed processes.

\section{Control of Thermal Process}

As an example let us consider the discrete-time control of an infinite-dimensional plant governed by the heat equation

$\frac{\partial Q(y, t)}{\partial t}=\frac{\partial}{\partial y}\left[r(y) \frac{\partial Q(y, t)}{\partial y}\right]+u(y, t)$,

$t>0, \quad 0<y<1$,

with boundary and initial conditions

$\frac{\partial Q}{\partial y}(0, t)=\frac{\partial Q}{\partial y}(1, t)=0, Q(y, 0)=Q_{0}(y)$.

Equation (9) describes the temperature field $Q(y, t)$ of a one-dimensional bar with distributed in space heat conductivity coefficient $r(y)$, heat-isolated ends and distributed heat source $u(y, t)$ as the control. If the solution $Q(y, t)$ is interpreted as a function of time with values in the space of double differentiable functions, then (9) can be viewed as an equation analogous to (1) in 
Banach space $L_{2}(0,1)$ with operator $A=\frac{\partial}{\partial y}\left[r(y) \frac{\partial(.)}{\partial y}\right]$ and $B=1$.

Denote eigenfunctions and eigenvalues of operator $A$ as $Y_{n}(y)$ and $\lambda_{n}, \quad n=(1, \infty)$ :

$\frac{\partial}{\partial y}\left[r(y) \frac{\partial Y_{n}(y)}{\partial y}\right]=\lambda_{n} Y_{n}(y)$,

$\partial Y_{n}(0) / \partial y=\partial Y_{n}(1) / \partial y=0$.

For heat equations $\lambda_{n}<0$ and $\lambda_{n} \rightarrow-\infty$ [11]. For example, if $r(y)$ is constant and equal to 1 , then

$$
Y_{n}(y)=\cos (n \pi y), \quad \lambda_{n}=-(n \pi)^{2}
$$

The solution to (9) is of form

$$
\begin{aligned}
Q(y, t)= & U(t) Q_{0}(y)+\int_{0} U(t-\tau) u(y, \tau) d \tau \\
= & \sum_{n=1}^{\infty} e^{\lambda_{n} t}\left[\int_{0} Q_{0}(\xi) Y_{n}(\xi) d \xi\right] Y_{n}(y) \\
& +\sum_{n=1}^{\infty}\left[\int_{0} e^{\lambda_{n}(t-\tau)} \times\right. \\
& \left.\left(\int_{0} u(\xi, \tau) Y_{n}(\xi) d \xi\right) d \tau\right] Y_{n}(y) .
\end{aligned}
$$

Bearing in mind that

$$
\begin{aligned}
& Q_{0}(y)=\sum_{n=1}^{\infty} q_{n}(0) Y_{n}(y) \\
& q_{n}(0)=2 \int_{0}^{1} Q_{0}(\xi) Y_{n}(\xi) d \xi \\
& u(y, t)=\sum_{n=1}^{\infty} u_{n}(t) Y_{n}(y) \\
& u_{n}(t)=2 \int_{0}^{1} u(\xi, t) Y_{n}(\xi) d \xi
\end{aligned}
$$

the validity of (12) as a solution to (10) may be checked by direct substitution.

Suppose that the control is a discrete-time function of $y$, i.e. $u(k)=u(y, k)$ for $\delta k \leq t<\delta(k+1)$. Then

$$
\begin{aligned}
Q(y, k+1) & =\sum_{n=1}^{\infty}\left[e^{\lambda_{n} \delta} q_{n}(k)\right. \\
& \left.+\left(\int_{0}^{\delta} e^{\lambda_{n}(\delta-\tau)} d \tau\right) u_{n}(k)\right] Y_{n}(y),
\end{aligned}
$$

where $q_{n}(k)$ and $u_{n}(k)$ may be found similarly to (13).

Following to the discrete-time sliding mode control methodology, the equivalent control should be found such that the manifold $Q(y)=0$ is reached after one step and then state trajectories are confined to this manifold. The distributed equivalent control may be found from (13) and (14)

$u_{e q}(y, k)=\sum_{n=1}^{\infty} u_{n, e q}(k) Y_{n}(y)$

where

$u_{n, e q}(k)=-\frac{\lambda_{n}}{e^{-\lambda_{n} \delta}-1} q_{n}(k)$.

The series (15) with finite values of $q_{n}(k)$ converges but $u_{e q}(y, k)$ may take high values with a small sampling interval $\delta$ and exceed the constraints posed on the control

$\|u\|_{L_{2}}=\left[\sum_{n=1}^{\infty} u_{n}^{2}\right]^{1 / 2} \leq u_{0}$.

Then similarly to (8), the control function (15), (16) is modified such that it always belongs to admissible domain (17), i.e.

$u_{n}(k)= \begin{cases}u_{n, e q} & \text { if }\left\|u_{e q}\right\| \leq u_{0} \\ \frac{\lambda_{n}}{e^{-\lambda_{n} \delta}-1} q_{n}(k) \frac{u_{0}}{\left\|u_{e q}\right\|} & \text { if }\left\|u_{e q}\right\|>u_{0} .\end{cases}$

If the equivalent control is beyond the admissible domain, $\left\|u_{e q}\right\|>u_{0}$, then

$$
\begin{aligned}
q_{n}(k+1) & =e^{\lambda_{n} \delta} q_{n}(k)+\left(\int e^{\lambda_{n}(\delta-\tau)} d \tau\right) u_{n}(k) \\
& =e^{\lambda_{n} \delta}\left[1-\frac{u_{0}}{\left\|u_{e q}\right\|}\right] q_{n}(k)
\end{aligned}
$$


This equation along with (16) indicates that $q_{n}(k)$ and $\left\|u_{e q}(k)\right\|$ converge to zero with $k \rightarrow \infty$. Hence after a finite number of steps $N$, the inequality $\left\|u_{e q}\right\| \leq u_{0}$ holds, and sliding mode exists on the manifold $Q(y, k)=0$ for $k>N$.

The distributed discrete-time sliding mode control may be designed as in (13):

$u(y, k)=\sum_{n=1}^{\infty} u_{n}(k) Y_{n}(y)$

with $u_{n}(k)$ in compliance with (18).

It is of interest to note that sliding mode is enforced with control as a continuous state function in contrast to continuous time systems with discontinuous control action.

\section{Adaptive Control}

Implementation of control (18), (19) needs knowledge of function $r(y)$ for finding eigenfunctions $Y_{n}(y)$ and eigenvalues $\lambda_{n}$ of linear operator $\frac{\partial}{\partial y}\left[r(y) \frac{\partial(.)}{\partial y}\right]$.

First we will demonstrate the method of deriving $r(y)$ based on a continuous-time model of the plant with sliding modes. Then a discrete-time approximation will be given for identification of this function.

The model equation with an adjustable distrubuted parameter is of form

$$
\frac{\partial \hat{Q}(y, t)}{\partial t}=\frac{\partial}{\partial y}\left[\hat{r}(y) \frac{\partial \hat{Q}(y, t)}{\partial y}\right]+u(y, t)+v[\bar{Q}(y, t)],
$$$$
t>0, \quad 0<y<1
$$

with boundary and initial conditions

$$
\begin{aligned}
& \frac{\partial \hat{Q}}{\partial y}(0, t)=\frac{\partial \hat{Q}}{\partial y}(1, t)=0 \\
& \hat{Q}(y, 0)=\hat{Q}_{0}(y) \\
& \text { and } \\
& \hat{Q}(y, t) \text { being the model state, }
\end{aligned}
$$

$\bar{Q}(y, t)=\hat{Q}(y, t)-Q(y, t)$

is the mismatch between the model and plant states, $\hat{r}(y, t)$ is the estimate of $r(y), \hat{r}(y, t)>0$,

The model input $v[\bar{Q}(y, t)]$ should be selected such that the mismatch $\bar{Q}(y, t)$ is reduced to zero. This function will depend on the unknown distributed parameter $r(y)$ which is needed for control (18), (19).

Subtracting (9) from (20) yields

$$
\frac{\partial \bar{Q}}{\partial t}=\frac{\partial}{\partial y}\left(\hat{r} \frac{\partial \bar{Q}}{\partial y}\right)+\frac{\partial}{\partial y}\left(\bar{r} \frac{\partial Q}{\partial y}\right)+v(\bar{Q})
$$

where $\bar{r}=\hat{r}-r$ is the estimation error.

Let $v$ be a discontinuous function of mismatch $\bar{Q}$

$$
v(\bar{Q})=-v_{0} \operatorname{sign}(\bar{Q}),
$$

where $v_{0}$ is a positive constant value.

$$
v_{0}>\left|\frac{\partial}{\partial y}\left(\bar{r} \frac{\partial Q}{\partial y}\right)\right| .
$$

(Note that function $Q(y, t)$ depends neither on $\hat{Q}(y, t)$ nor on $v$ in mismatch equation (21)).

To prove the convergence of mismatch $\bar{Q}(y, t)$ to zero calculate the time derivative of Lyapunov functional candidate

$$
V(t)=0.5 \int_{0}^{1} \bar{Q}^{2} d y
$$

on system (21) trajectories

$$
\begin{aligned}
& \dot{V}=\int \bar{Q}\left[\frac{\partial}{\partial y}\left(\hat{r} \frac{\partial \bar{Q}}{\partial y}\right)+\frac{\partial}{\partial y}\left(\bar{r} \frac{\partial Q}{\partial y}\right)+v(\bar{Q})\right] d y \\
& =\left.\left[Q\left(\hat{r} \frac{\partial \bar{Q}}{\partial y}\right)\right]\right|_{0} ^{1}-\int_{0}^{(}\left(\frac{\partial \bar{Q}}{\partial y}\right)^{2} \hat{r} d y \\
& +\int\left(\bar{Q} \frac{\partial}{\partial y}\left(\bar{r} \frac{\partial Q}{\partial y}\right)-v_{0}|\bar{Q}|\right) d y \text {. }
\end{aligned}
$$

Since the boundary values of $\partial \bar{Q} / \partial y$ are equal to zero, $\hat{r}(y, t)>0$, the time derivative $\dot{V}$ is negative by virtue of the condition (23). Lyapunov functional 
$V=0.5\|\bar{Q}\|^{2}$, hence the norm of mismatch $\bar{Q}$ tends to zero.

In steady state mode the argument of discontinuous function $v$ is equal to zero. This means that sliding mode occurs in the system.

The identification process is governed by the equation

$\frac{\partial \hat{r}(y, t)}{\partial t}=\gamma \frac{\partial Q}{\partial y} \int_{b}^{y} v[\bar{Q}(\xi, t)] d \xi$

with $\hat{r}(y .0)>0$ and $\gamma>0$. Since $r(y)$ does not depend on time

$$
\frac{\partial \vec{r}(y, t)}{\partial t}=\gamma \frac{\partial Q}{\partial y} \int_{0}^{y} v[\bar{Q}(\xi, t)] d \xi .
$$

To derive the motion equations of the system in sliding mode discontinuous function $v$ should be replaced by the equivalent control $v_{e q}[4]$, which is the solution to the equation $\dot{\bar{Q}}=0$ with $\bar{Q}=0$. As it follows from (21)

$v_{e q}=-\frac{\partial}{\partial y}\left(\bar{r} \frac{\partial Q}{\partial y}\right)$.

Substituting this function into (23) results in

$$
\frac{\partial \bar{r}}{\partial t}=-\bar{r}\left(\frac{\partial Q}{\partial y}\right)^{2}
$$

If $\int_{0}^{\infty}\left(\frac{\partial Q}{\partial y}\right)^{2} d t$ is unbounded, then estimation error $\vec{r}(y, t)$ tends to zero and

$\lim \hat{r}(y, t)=r(y)$ with $t \rightarrow \infty$.

The discrete-time approximation of the identification algorithm (20), (22), (23), (24) is of form

$$
\begin{aligned}
\hat{Q}_{k+1}(y)=\hat{Q}_{k}(y)+\delta\left\{\frac { \partial } { \partial y } \left[\hat{r}_{k}(y)\right.\right. & \left.\frac{\partial \hat{Q}_{k}(y)}{\partial y}\right]+u_{k}(y) \\
& \left.+v\left[\bar{Q}_{k}(y)\right]\right\}
\end{aligned}
$$

$\hat{r}_{k}(y)=\hat{r}_{k-1}(y)+\delta \gamma \frac{\partial Q_{k}}{\partial y} \int_{0}^{\gamma} v\left[\bar{Q}_{k}(\xi)\right] d \xi$.

To alleviate chattering, discontinuous function (22) is replaced by a saturation function [9]

$$
v\left(\overline{Q_{k}}\right)=\left\{\begin{array}{cc}
-v_{0} \operatorname{sign}\left(\bar{Q}_{k}\right) & \text { if } 1 / \delta\left|\bar{Q}_{k}\right|>v_{0} \\
-1 / \delta \bar{Q}_{k} & \text { otherwise }
\end{array} .\right.
$$

The estimated value $\hat{r}(y)$ enables one to find the eigenfunctions and eigenvalues and implement control algorithm (18), (19) resulting in the ideal sliding mode with the state trajectories in the manifold $s=0$.

\section{References}

1. V.I. Utkin,"Sliding Modes in Control and Optimization", Springer Verlag, Berlin, 1992.

2. "Variable Structure Control for Robotics and Aerospace Applications", K-K. D. Young, Ed., Elsevier Science Publishers B.V.,Amsterdam, 1993.

3. R. A. DeCarlo, S.H. Zak, G.P. Matthew, Variable Structure Control of Nonlinear Multivariable Systems: A Tutorial, Proceedings of the IEE, Vol.76. No.3, pp. 212-232, March 1988.

4. V.I. Utkin and Yu.V. Orlov, Sliding Mode Control in Infinite-Dimensional Systems. Automatica, No 6, pp. 753-756, 1987.

5. S.E. Rebai, A.S.I. Zinober, Stabilization of Distributed Parameter Systems. International Journal of Control, Vol.57, No.5, pp.1167-1176, May 1993.

6. H. Sira-Ramirez, Distributed Sliding Mode Control in Systems Described by Quasilinear Partial Differential Equations. Systems and Control Letters 13, North-Holland, pp. 177-181, 1989.

7. S.V. Drakunov and V.I. Utkin, Sliding Mode Control in Dynamic Systems. International Journal of Control, Vol.55,, No 4, pp. 1029-1037, 1992.

8. S.V. Drakunov and V.I. Utkin, On Discrete-Time Sliding Modes, Preprint of Interantional Symposium IFAC on Nonlinear Control Systems Design, June 14-16, 1989, Capry, Italy.

9. V.I. Utkin, Sliding Mode Control in Discrete-Time and Difference Systems in book "Variable Structure and Lyapunov Control", A.S. Zinober, Ed., Springer Verlag, London, pp.87-107, 1993.

10. R.F. Curtain, A.J. Pritchard, "Infinite-dimensional Linear Systems Theory", in Lecture Notes in Control and Information Science, Springer Verlag, Berlin, 1978.

11. D. Henry, "Partial Differential Equations", Holt, Reinhart, and Winston, New York, 1969. 